\title{
Two-Pomeron eikonal approximation for the high-energy elastic diffractive scattering of nucleons
}

\author{
A. A. Godizov $\oplus^{*}$ \\ A.A. Logunov Institute for High Energy Physics, NRC “Kurchatov Institute”, \\ 142281 Protvino, Russia
}

(Received 23 July 2019; accepted 6 April 2020; published 22 April 2020)

\begin{abstract}
We demonstrate that the elastic diffractive scattering of nucleons at collision energies higher than $540 \mathrm{GeV}$ and transferred momenta lower than $2 \mathrm{GeV}$, including the Coulomb-nuclear interference region, can be described in the framework of a very simple Regge-eikonal model where the eikonal is just a sum of two supercritical Regge pole terms. The predictive value of the proposed approximation is verified.
\end{abstract}

DOI: 10.1103/PhysRevD.101.074028

\section{INTRODUCTION}

During the last several decades, perturbative quantum chromodynamics (pQCD) confirmed its usefulness many times as a powerful theoretical tool in the sector of high energies and high transferred momenta of strongly interacting particles. However, at present, a very large part of hadron physics cannot be treated in the framework of this quantum-field model. Particularly, to quantitatively describe the elastic diffractive scattering (EDS) of nucleons at high values of the collision energy and low values of the transferred momentum, we have to use phenomenological models which are not based on any analytic approximations within QCD. The absence of a direct connection between these models and the fundamental theory of strong interaction very often reduces their predictive power. As a result, in 2011, many hadron diffraction models nicely described the available experimental data on the proton-(anti)proton EDS in the energy range from the ISR to the Tevatron (with the collision energy increasing tens of times) but failed to reproduce the behavior of the $p p$ angular distribution in the region of the diffraction dip and nonforward peak at the LHC [1]. The discrepancy between the model predictions and the experimental data is so huge that it could lead to a justified conclusion about our total misunderstanding of the physical mechanisms of hadron diffraction (for a detailed discussion, see the minireview in [2]).

\footnotetext{
anton.godizov@gmail.com
}

Published by the American Physical Society under the terms of the Creative Commons Attribution 4.0 International license. Further distribution of this work must maintain attribution to the author(s) and the published article's title, journal citation, and DOI. Funded by SCOAP ${ }^{3}$.
Nonetheless, the fraction of EDS events in the total number of events at high-energy hadron colliders is so significant ( $\sim 25 \%$ at the LHC) that we still need to develop reliable phenomenological approaches which could help properly interpret the results of the measurements in the high-energy hadron diffraction region. Of course, any model should be verifiable (and, certainly, discriminable) on the available and forthcoming experimental data.

All the modern models of EDS could be divided into two groups: those based on Regge theory [3] and the nonReggeon models. The references to various Reggeon and non-Reggeon phenomenological schemes can be found in a recent review [4] and in the RPP [5]. The aim of this paper is to demonstrate the predictive value and reliability of the two-Pomeron eikonal approximation proposed earlier in [6]. It will be done via application to the new elastic scattering data sets produced recently by the TOTEM Collaboration [7].

\section{THE MODEL}

\section{A. The strong interaction subamplitude}

The physical content of the two-Pomeron eikonal approximation is very simple and transparent. In the kinematic range wherein the Coulomb interaction may be considered negligible, the eikonal for the high-energy EDS of nucleons can be represented as a sum of two supercritical Regge pole terms. The first term corresponds to exchange by the socalled soft Pomeron (SP). This interaction is the basic cause of the visible growth of the proton-proton total and elastic cross sections at high energies. The second term corresponds to the exchange by the hard Pomeron (HP), also known as the BFKL Pomeron. A detailed discussion of the considered model can be found in [6]. Below we give a recipe for calculation of the EDS angular distributions in the framework of this approximation: 


$$
\begin{aligned}
\frac{d \sigma}{d t}= & \frac{\left|T_{N}(s, t)\right|^{2}}{16 \pi s^{2}}, \quad T_{N}(s, t)=4 \pi s \int_{0}^{\infty} d\left(b^{2}\right) J_{0}(b \sqrt{-t}) \frac{e^{2 i \delta_{N}(s, b)}-1}{2 i}, \\
\delta_{N}(s, b)= & \frac{1}{16 \pi s} \int_{0}^{\infty} d(-t) J_{0}(b \sqrt{-t})\left[\delta_{\mathrm{SP}}(s, t)+\delta_{\mathrm{HP}}(s, t)\right]=\frac{1}{16 \pi s} \int_{0}^{\infty} d(-t) J_{0}(b \sqrt{-t}) \\
& \times\left[\xi_{+}\left(\alpha_{\mathrm{SP}}(t)\right) g_{\mathrm{SP}}^{2}(t) \pi \alpha_{\mathrm{SP}}^{\prime}(t)\left(\frac{s}{2 s_{0}}\right)^{\alpha_{\mathrm{SP}}(t)}+\xi_{+}\left(\alpha_{\mathrm{HP}}(0)\right) \beta_{\mathrm{HP}}(t)\left(\frac{s}{2 s_{0}}\right)^{\alpha_{\mathrm{HP}}(0)}\right],
\end{aligned}
$$

where $s$ and $t$ are the Mandelstam variables, $b$ is the impact parameter, $s_{0}=1 \mathrm{GeV}^{2}, J_{0}(x)$ is the Bessel function, $\alpha_{\mathrm{SP}}(t)$ is the SP Regge trajectory, $g_{\mathrm{SP}}(t)$ is the SP coupling to the nucleon, $\alpha_{\mathrm{HP}}(0)$ is the intercept of the HP Regge trajectory [it was argued in [6] why the $t$ dependence of $\alpha_{\mathrm{HP}}(t)$ is negligible in the region of EDS], $\beta_{\mathrm{HP}}(t) \equiv g_{\mathrm{HP}}^{2}(t) \pi \alpha_{\mathrm{HP}}^{\prime}(t)$ is the HP Regge residue, $\xi_{+}(\alpha)=\left(i+\tan \frac{\pi(\alpha-1)}{2}\right)$ are the signature factors for even Reggeons, $\delta_{N}$ is the eikonal (Born amplitude), and $T_{N}$ is the full amplitude related to a strong interaction.

The HP intercept can be extracted from the data on the proton unpolarized structure function $F_{2}^{p}\left(x, Q^{2}\right)$ [8] at high values of the incoming photon virtuality $Q^{2}$ and low values of the Bjorken scaling variable $x: \alpha_{\mathrm{HP}}(0) \approx 1.32$ [9]. In the region of low negative $t$, the unknown functions $\alpha_{\mathrm{SP}}(t)$, $g_{\mathrm{SP}}(t)$, and $\beta_{\mathrm{HP}}(t)$ may be approximated with the help of the following simple test parametrizations $[6,10]$ :

$\alpha_{\mathrm{SP}}(t)=1+\frac{\alpha_{\mathrm{SP}}(0)-1}{1-\frac{t}{\tau_{a}}}, \quad g_{\mathrm{SP}}(t)=\frac{g_{\mathrm{SP}}(0)}{\left(1-a_{g} t\right)^{2}}$,

$\beta_{\mathrm{HP}}(t)=\beta_{\mathrm{HP}}(0) e^{b t}$,

where the free parameters take on the values presented in Table I.

Usage of essentially nonlinear parametrization for the SP Regge trajectory distinguishes the proposed model from other models [11-14] which exploit linear Regge trajectories. The main reason to presume a strongly nonlinear behavior of $\alpha_{\mathrm{SP}}(t)$ at $t<0$ is based on the assumption that in the region of extremely high transfers, $\sqrt{-t} \gg 1 \mathrm{TeV}$, any exchange by the SP turns into the exchange by two

TABLE I. The parameter values for (2) obtained earlier $[6,10]$ via fitting to the elastic scattering data in the collision energy range $546 \mathrm{GeV} \leq \sqrt{s} \leq 7 \mathrm{TeV}$.

\begin{tabular}{ll}
\hline \hline Parameter & \multicolumn{1}{c}{ Value } \\
\hline$\alpha_{\mathrm{SP}}(0)-1$ & 0.109 \\
$\tau_{a}$ & $0.535 \mathrm{GeV}^{2}$ \\
$g_{\mathrm{SP}}(0)$ & $13.8 \mathrm{GeV}^{-2}$ \\
$a_{g}$ & $0.23 \mathrm{GeV}^{-2}$ \\
$\beta_{\mathrm{HP}}(0)$ & 0.08 \\
$b$ & $1.5 \mathrm{GeV}^{-2}$ \\
$\alpha_{\mathrm{HP}}(0)-1$ & $0.32($ fixed) \\
\hline \hline
\end{tabular}

gluons. At asymptotically high energies, such perturbative amplitudes behave as $T^{(g g)}(s, t) \sim T^{(\gamma \gamma)}(s, t) \sim s$ [15-17], which implies the following asymptotic relation: $\lim _{t \rightarrow-\infty} \alpha_{\mathrm{SP}}(t)=1$. A detailed discussion of why any hadron Regge trajectory must exhibit a nonlinear behavior at $t<0$ and how such a nonlinearity may be consistent with approximately linear behavior in the resonance region, $t>0$, can be found in [18]. Unfortunately, at its current stage of development, QCD does not allow direct calculation of $\alpha_{\mathrm{SP}}(t)$, or of $g_{\mathrm{SP}}(t)$ and $\beta_{\mathrm{HP}}(t)$, in the nonperturbative region. As a consequence, one has to use some test parametrizations to approximate the unknown functions $\alpha_{\mathrm{SP}}(t), g_{\mathrm{SP}}(t)$, and $\beta_{\mathrm{HP}}(t)$ in the range of low negative $t$. In view of this fact, we emphasize that expressions (2) are just phenomenological. In particular, the true Regge trajectories of both the Pomerons have branching points at the two-pion threshold (for details, see chapter 3 in [3]) and have no poles on the physical sheet. Therefore, parametrizations (2) must be treated as phenomenological nonanalytic approximations to the true functions $\alpha_{\mathrm{SP}}(t), g_{\mathrm{SP}}(t)$, and $\beta_{\mathrm{HP}}(t)$ in the region $t<0$ only. They are absolutely invalid in the region $\operatorname{Re} t>0$. However, exploitation of such parametrizations is quite justified due to the above-mentioned fact that the exact analytic behavior of $\alpha_{\mathrm{SP}}(t), g_{\mathrm{SP}}(t)$, and $\beta_{\mathrm{HP}}(t)$ is still unknown.

\section{B. Impact of electromagnetic interaction}

To describe the EDS of protons in the region of Coulomb-nuclear interference, we need to take account of electromagnetic interaction.

In the framework of the eikonal approach, the full amplitude of the proton-(anti)proton EDS in the coordinate representation has the following structure:

$$
\begin{aligned}
T(s, b) & =\frac{e^{2 i\left(\delta_{C}(s, b)+\delta_{N}(s, b)\right)}-1}{2 i} \\
& =T_{N}(s, b)+\delta_{C}(s, b)+2 i T_{N}(s, b) \delta_{C}(s, b)+O\left(\alpha_{e}^{2}\right),
\end{aligned}
$$

where $\delta_{C}(s, b) \sim \alpha_{e}$ is the tree-level subamplitude of electromagnetic interaction, and $\alpha_{e}$ is the fine structure constant. 

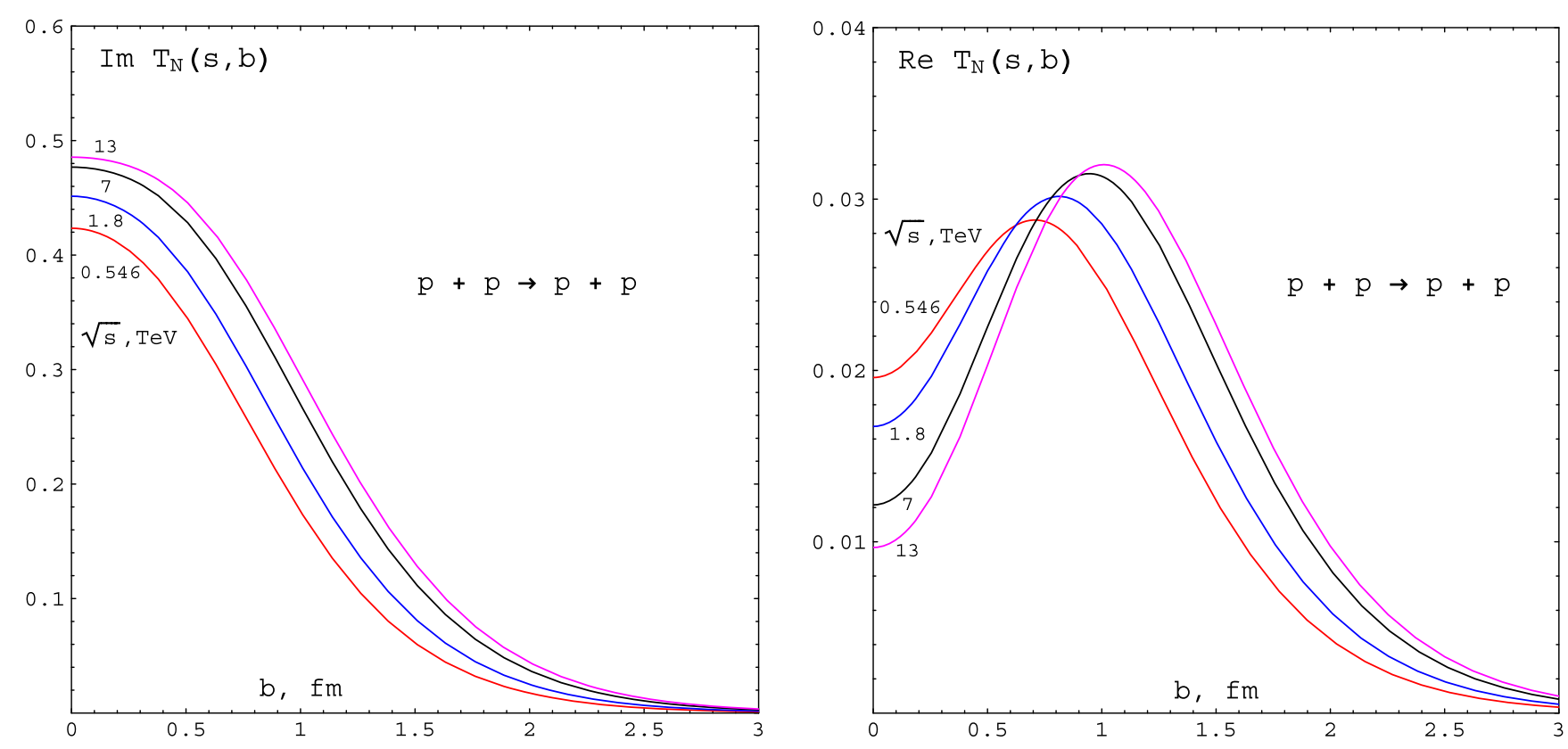

FIG. 1. The imaginary and real parts of the strong interaction subamplitude $T_{N}(s, b)$ at various values of the collision energy.

At large enough values of the impact parameter, electromagnetic interaction dominates: $\left|\delta_{C}(s, b)\right| \gg\left|T_{N}(s, b)\right|$ and, hence, $T(s, b) \approx \delta_{C}(s, b)$. At small values of $b$, the electromagnetic interaction of protons can be ignored: $T(s, b) \approx T_{N}(s, b)$. In the range $3 \mathrm{fm}<b<10 \mathrm{fm}$, wherein $\left|\delta_{C}(s, b)\right| \sim\left|T_{N}(s, b)\right|$, we may ignore the third term in (3) because $\left|T_{N}(s, b)\right| \ll 1$ in this region [the fast decrease of $\left|T_{N}(s, b)\right|$ can be seen in Fig. 1], and, consequently, this term is negligible compared with the first two.

Thus, finally, we come to the leading approximation of the full (electromagnetic + strong) amplitude in the entire kinematic range of EDS:

$$
\begin{aligned}
T(s, b) & \approx \delta_{C}(s, b)+T_{N}(s, b), \\
\Rightarrow \quad T(s, t) & \approx \delta_{C}(s, t)+T_{N}(s, t) .
\end{aligned}
$$

In other words, we neglect the Coulomb-nuclear interference at the amplitude level.

It should be noted here that such a negligibility is a model-dependent effect. In the framework of many models, the corresponding terms are expected to yield a significant contribution to the full amplitude (for a detailed discussion, see [19] and references therein).

In the transferred momentum range $0<\sqrt{-t}<2 \mathrm{GeV}$, the Coulomb term can be approximated by a simple expression

$$
\delta_{C}(s, t)= \pm \frac{8 \pi s \alpha_{e}}{t} F_{E}^{2}(t)
$$

where $F_{E}(t)=\left(1-\frac{t}{0.71 \mathrm{GeV}^{2}}\right)^{-2}$ is the dipole electric form factor of the proton.

\section{VERIFICATION OF THE MODEL}

\section{A. Model predictions versus the newest experimental data}

To check the model efficiency, we need to compare the model predictions with the new data [7] on the protonproton EDS at $\sqrt{s}=13 \mathrm{TeV}$. The results of such a verification (without any refitting of the model parameters) are presented in Fig. 2.

The data description quality in terms of the method of least squares is $\chi^{2}=1796$ over 428 points (the description quality in the range $\sqrt{-t}<0.1 \mathrm{GeV}$ is $\chi^{2}=18$ over 25 points). Hereby, we observe a rather weak deviation of the model curve from the experimental data. ${ }^{1}$

The model predictions for the $p p$ total cross section and for $\rho=\frac{\operatorname{Re} T_{N}(s, 0)}{\operatorname{Im} T_{N}(s, 0)}$ at $\sqrt{s}=13 \mathrm{TeV}$ are $\sigma_{\mathrm{tot}}^{\operatorname{model}}(13 \mathrm{TeV})$ $\approx 109.4 \mathrm{mb}$ and $\rho^{\text {model }}(13 \mathrm{TeV}) \approx 0.125$, while the corresponding measured values are $\sigma_{\text {tot }}(13 \mathrm{TeV})=(110.5 \pm$ 2.4) $\mathrm{mb}$ and $\rho(13 \mathrm{TeV})=0.10 \pm 0.01$ [7]. It should be noted here that extraction of these quantities from the experimental angular distributions is a strongly modeldependent procedure.

Concerning the sensitivity of the considered model with respect to the fixed value of the HP intercept, it should be noted here that if we put $\alpha_{\mathrm{HP}}(0)=1.44$ and fit $\beta_{\mathrm{HP}}(t)$ to the

\footnotetext{
${ }^{1}$ It should be pointed out here that the exploited parametrizations (2) for $\alpha_{\mathrm{SP}}(t)$ and $g_{\mathrm{SP}}(t)$ allow one to obtain a satisfactory description of the exclusive photoproduction of light vector mesons [20] and the proton single diffractive dissociation [21] in the kinematic ranges where the impact of the hard Pomeron and secondary Reggeon exchanges is negligible compared with the experimental uncertainties.
} 

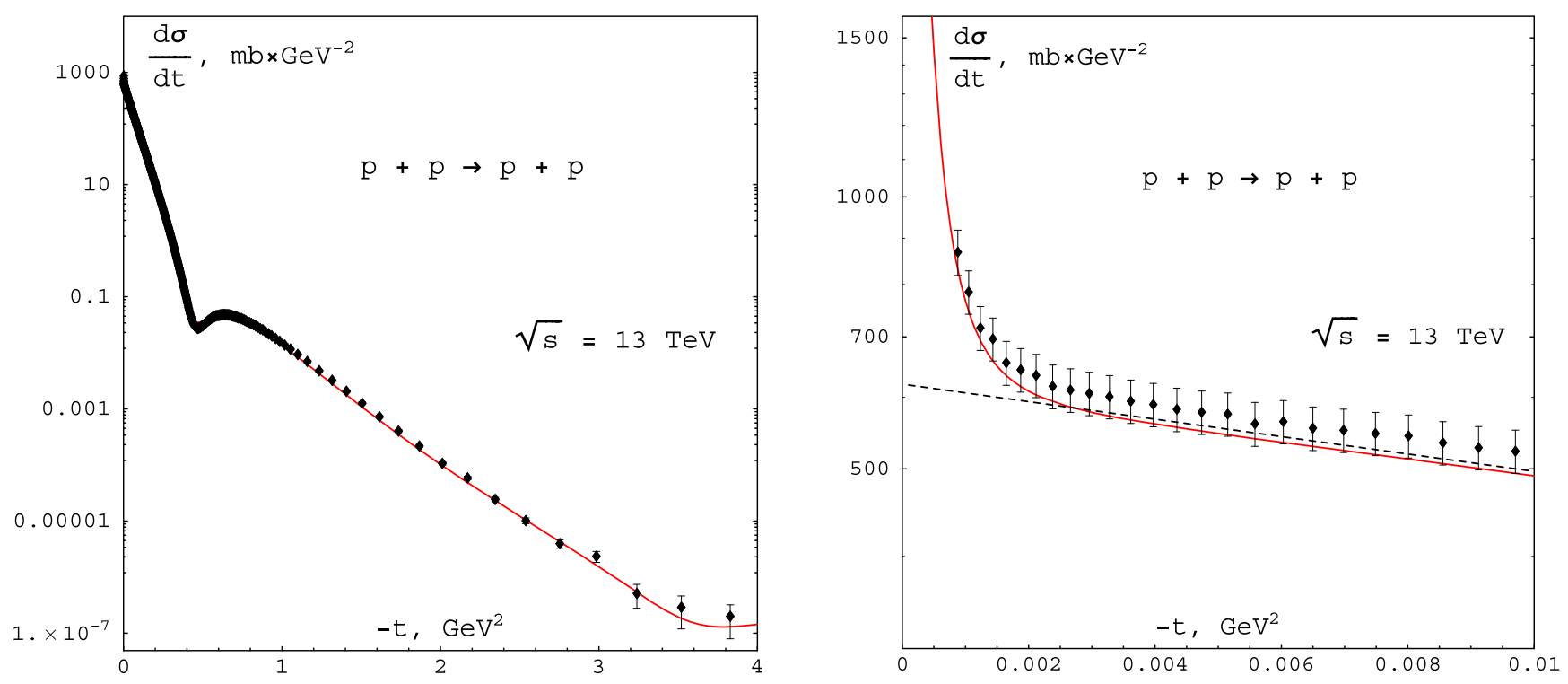

FIG. 2. The predictions of the model [6] in the case $\alpha_{\mathrm{HP}}(0)-1=0.32$ versus the TOTEM data at $\sqrt{s}=13$ TeV [7]. The dashed line corresponds to the approximation $\delta_{C}(s, t)=0$.

data at $7 \mathrm{TeV}$ of the collision energy (see Fig. 2 in [6]), then, at $13 \mathrm{TeV}$, we obtain the prediction for the $p p$ angular distribution presented in Fig. 3. As we can see, at such a value of the HP intercept, the model demonstrates a noticeable overestimation of the observed $d \sigma / d t$ in the region $\sqrt{-t}>1.5 \mathrm{GeV}$. In other words, the proposed phenomenological scheme is quite sensitive to the value of $\alpha_{\mathrm{HP}}(0)$, and thus, we may interpret the result presented in Fig. 2 as some model-dependent confirmation of the fact that the HP in the high-energy EDS of nucleons is the same supercritical Reggeon that governs the low- $x$ behavior of the proton unpolarized structure function $F_{2}^{p}\left(x, Q^{2}\right)$ at high

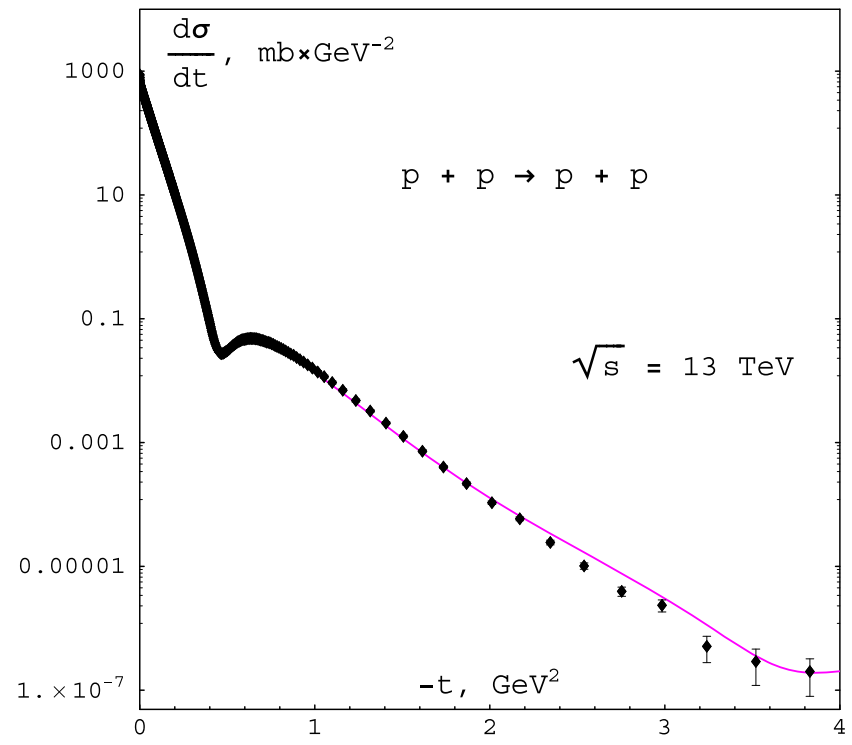

FIG. 3. The prediction of the model [6] in the case $\alpha_{\mathrm{HP}}(0)-$ $1=0.44$ versus the TOTEM data at $\sqrt{s}=13 \mathrm{TeV}$ [7]. values of the incoming photon virtuality $Q^{2}$ in deeply inelastic scattering of leptons on protons.

\section{B. 3.2. Results of refitting}

The next step is to refit the model parameters to the enlarged set of data, exploiting the same parametrizations (2)

TABLE II. The parameter values for (2) obtained via fitting to the EDS data in the entire kinematic range $\{546 \mathrm{GeV} \leq$ $\sqrt{s} \leq 13 \mathrm{TeV}, \sqrt{-t}<2 \mathrm{GeV}\}$.

\begin{tabular}{ll}
\hline \hline Parameter & \multicolumn{1}{c}{ Value } \\
\hline$\alpha_{\mathrm{SP}}(0)-1$ & 0.1055 \\
$\tau_{a}$ & $0.572 \mathrm{GeV}^{2}$ \\
$g_{\mathrm{SP}}(0)$ & $14.7 \mathrm{GeV}^{-2}$ \\
$a_{g}$ & $0.32 \mathrm{GeV}^{-2}$ \\
$\beta_{\mathrm{HP}}(0)$ & 0.108 \\
$b$ & $1.55 \mathrm{GeV}^{-2}$ \\
$\alpha_{\mathrm{HP}}(0)-1$ & 0.32 (fixed) \\
\hline \hline
\end{tabular}

TABLE III. The quality of the description of the data [1,7,22] on the EDS angular distributions.

\begin{tabular}{lcr}
\hline \hline$\sqrt{s}, \mathrm{GeV}$ & Number of points & \multicolumn{1}{c}{$\chi^{2}$} \\
\hline $546(\bar{p} p ; \mathrm{UA} 4)$ & 187 & 264 \\
$630(\bar{p} p ; \mathrm{UA} 4)$ & 19 & 34 \\
$1800(\bar{p} p ; \mathrm{E} 710)$ & 51 & 19 \\
$1960(\bar{p} p ; \mathrm{D} 0)$ & 17 & 24 \\
$2760(p p ;$ TOTEM $)$ & 63 & 180 \\
$7000(p p ;$ TOTEM, ATLAS $)$ & 205 & 323 \\
$8000(p p ;$ TOTEM, ATLAS $)$ & 69 & 159 \\
$13000(p p ;$ TOTEM $)$ & 428 & 982 \\
Total & 1039 & 1985 \\
\hline \hline
\end{tabular}



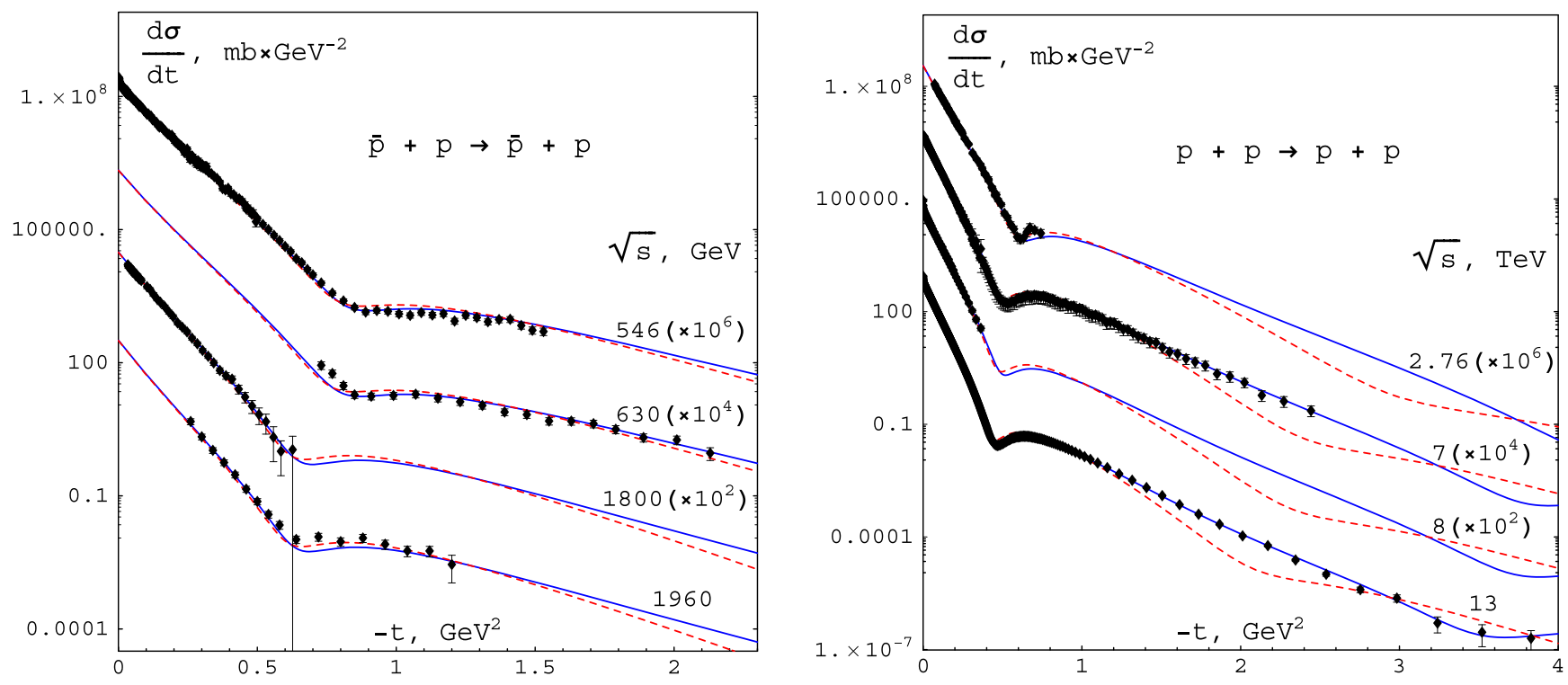

FIG. 4. The differential cross sections of nucleon-nucleon EDS at ultrahigh energies. The dashed lines correspond to the one-Pomeron eikonal approximation wherein the HP exchange contribution to the eikonal is ignored.

for the unknown functions $\alpha_{\mathrm{SP}}(t), g_{\mathrm{SP}}(t)$, and $\beta_{\mathrm{HP}}(t)$ as in [6] (this is necessary for improvement of the description quality). The results are presented in Tables II and III and Fig. 4.

The main cause of the observed slight discrepancy between the model issues and the experimental data is, certainly, the stiffness of the parametrizations used for the SP Regge trajectory and the SP coupling to the proton. More flexible parametrizations could result in a better description of the data, though one should keep in mind that the true analytic behavior of $\alpha_{\mathrm{SP}}(t), g_{\mathrm{SP}}(t)$, and $\beta_{\mathrm{HP}}(t)$ remains unknown. Nonetheless, the simplicity of test functions (2) makes them very attractive for usage in the region of low negative $t$, while the achieved quality of the description makes the model quite suitable for rough estimations and predictions of the nucleon-nucleon EDS observables at ultrahigh energies.

Another possible cause of the noticeable deviation of the model curves from the $\bar{p} p$ scattering data at $\sqrt{s}=$ $1.96 \mathrm{TeV}$ and $0.6 \mathrm{GeV}^{2}<-t<0.9 \mathrm{GeV}^{2}$ and, in the same $t$ interval, from the $p p$ scattering data at $\sqrt{s}=$ $2.76 \mathrm{TeV}$ is the impact of exchanges by the Odderon (the $C$-odd counterpartner of the SP). The Odderon exchange contribution is completely ignored in the framework of the considered two-Pomeron eikonal approximation. Although most scientists believe in the existence of such a supercritical Reggeon which gives different contributions into the $p p$ and $\bar{p} p$ interactions, the significance of the Odderon exchanges at available energies is still a subject of discussion. For example, in the most well-known model of elastic diffractive scattering [11], the influence of the
Odderon exchanges is considered to be negligible. On the contrary, the authors of Ref. [14] insist on the necessity to take account of the Odderon contribution. The scheme presented in [13] admits both of the variants. In any case, the Odderon impact on the angular distributions of the high-energy elastic scattering of nucleons seems to be a much finer effect than the impact of the HP exchanges. At the LHC energies, ignoring the HP contribution to the eikonal can lead to a catastrophic divergence between the model curves and the data in the region $-t>1.5 \mathrm{GeV}^{2}$ (see Fig. 4).

\section{CONCLUSION}

We conclude that the two-Pomeron eikonal approximation successfully confirmed its predictive value; thus, it can be considered as a simple and reliable phenomenological tool for a qualitative description of the nucleon-nucleon EDS at ultrahigh energies. In addition, we confirmed, in the framework of the proposed model, that the HP which has a crucial impact on the nucleon-nucleon diffraction pattern at the LHC energies and transferred momenta higher than $1 \mathrm{GeV}$ is the same supercritical Reggeon which governs the low- $x$ evolution of the proton unpolarized structure function $F_{2}^{p}\left(x, Q^{2}\right)$ in DIS at high values of the incoming photon virtuality. The test functions $\alpha_{\mathrm{SP}}(t)$ and $g_{\mathrm{SP}}(t)$ with the free parameter values fitted to the available data can be used in the framework of Reggeon models for more compound reactions, such as high-energy single diffractive dissociation of nucleons or central exclusive production of light neutral mesons. 
[1] TOTEM Collaboration, Europhys. Lett. 95, 41001 (2011); 101, 21002 (2013).

[2] A. A. Godizov, AIP Conf. Proc. 1523, 145 (2013).

[3] P. D. B. Collins, An Introduction to Regge Theory \& High Energy Physics (Cambridge University Press, Cambridge, England, 1977).

[4] L. Jenkovszky, R. Schicker, and I. Szanyi, Int. J. Mod. Phys. E 27, 1830005 (2018).

[5] M. Tanabashi et al. (Particle Data Group), Phys. Rev. D 98, 030001 (2018).

[6] A. A. Godizov, Phys. Rev. D 96, 034023 (2017).

[7] TOTEM Collaboration, Eur. Phys. J. C 79, 785 (2019); 79, 861 (2019).

[8] See www.desy.de/h1zeus/combined_results; H1 and ZEUS Collaborations, J. High Energy Phys. 01 (2010) 109.

[9] A. A. Godizov, Nucl. Phys. A927, 36 (2014).

[10] A. A. Godizov, Eur. Phys. J. C 75, 224 (2015).

[11] A. Donnachie and P. V. Landshoff, Phys. Lett. B 727, 500 (2013).

[12] O. V. Selyugin, Nucl. Phys. A959, 116 (2017).
[13] V. A. Khoze, A. D. Martin, and M. G. Ryskin, Phys. Lett. B 784, 192 (2018).

[14] E. Martynov and B. Nicolescu, Eur. Phys. J. C 79, 461 (2019).

[15] H. Cheng and T. T. Wu, Phys. Rev. Lett. 22, 666 (1969).

[16] P. D. B. Collins and P. J. Kearney, Z. Phys. C 22, 277 (1984).

[17] R. Kirschner and L. N. Lipatov, Z. Phys. C 45, 477 (1990).

[18] A. A. Godizov and V. A. Petrov, J. High Energy Phys. 07 (2007) 083.

[19] V. A. Petrov, Eur. Phys. J. C 78, 221 (2018).

[20] A. A. Godizov, Eur. Phys. J. C 76, 361 (2016).

[21] A. A. Godizov, Nucl. Phys. A955, 228 (2016).

[22] M. Bozzo et al. (UA4 Collaboration), Phys. Lett. B 147, 385 (1984); 155, 197 (1985); D. Bernard et al. (UA4 Collaboration), Phys. Lett. B 198, 583 (1987); 171, 142 (1986); N. Amos et al. (E-710 Collaboration), Phys. Lett. B 247, 127 (1990); D0 Collaboration, Phys. Rev. D 86, 012009 (2012); ATLAS Collaboration, Nucl. Phys. B889, 486 (2014); TOTEM Collaboration, Eur. Phys. J. C 76, 661 (2016); ATLAS Collaboration, Phys. Lett. B 761, 158 (2016); TOTEM Collaboration, Eur. Phys. J. C 80, 91 (2020). 